\title{
Direct Nucleosome Binding of Borealin Secures Chromosome Association and Function of the Chromosomal Passenger complex
}

\author{
M. A. Abad ${ }^{1}$, J. G. Ruppert ${ }^{1}$, L. Buzuk ${ }^{1}$, M. Wear ${ }^{1}$, J. Zou' ${ }^{1}$, K. M. Webb ${ }^{1}$, D. A. Kelly ${ }^{1}$, P. Voigt ${ }^{1}$, \\ J. Rappsilber ${ }^{1,2}$, W. C. Earnshaw ${ }^{1}$ and A. A. Jeyaprakash ${ }^{1 *}$ \\ ${ }^{1}$ Wellcome Centre for Cell Biology, University of Edinburgh, Edinburgh EH93BF, UK. \\ 2 Technical University, Berlin 13355, Germany. \\ *email: jeyaprakash.arulanandam@ed.ac.uk
}

\section{Summary}

Chromosome association of the Chromosomal Passenger Complex (CPC; consisting of Borealin, Survivin, INCENP and the Aurora B kinase) is essential to achieve error-free chromosome segregation during cell division. Hence, understanding the mechanisms driving the chromosome association of the CPC is of paramount importance. Here using a multifaceted approach, we show that the CPC binds nucleosomes through a multivalent interaction predominantly involving Borealin. Strikingly, Survivin, previously suggested to target the CPC to centromeres [1-3] failed to bind nucleosomes on its own and requires Borealin and INCENP for its binding. Disrupting Borealin-nucleosome interactions excluded the CPC from chromosomes and caused chromosome congression defects. We also show that Borealin-mediated chromosome association of the CPC is critical for Haspin- and Bub1mediated centromere enrichment of the CPC and works upstream of the latter. Our work thus establishes Borealin as a master regulator determining the chromosome association and function of the CPC.

\section{Introduction}

Chromosome segregation is a complex process involving numerous protein-protein and protein-DNA interactions tightly controlled by signaling networks consisting of kinases and phosphatases [4-6]. Aurora B kinase, the enzymatic core of the Chromosomal Passenger Complex $(\mathrm{CPC})$ is a key regulator essential for error-free chromosome segregation and 
functions by controlling multiple steps of cell division: chromosome condensation and cohesion, kinetochore-microtubule attachments, the spindle assembly checkpoint and cytokinesis (reviewed in $[4,7,8]$ ). The CPC is composed of Aurora B, INCENP, Borealin/Dasra and Survivin and can be divided into distinct localisation and kinase modules, linked by the central helical coil of INCENP. The localisation module (CPC_LM) consisting of Borealin, Survivin and the first 58 amino acids (aa) of INCENP controls the localisation of the CPC [9, 10]. The kinase module consists of Aurora B and the IN-box of INCENP, a well-conserved Cterminal region required for full activation of Aurora B kinase [11-13]. CPC function is tightly linked to its distinct localisation during different stages of cell division. During early stages of mitosis the CPC localises to chromosome arms where it influences chromosome condensation $[14,15]$. It subsequently concentrates at the inner centromere where it releases incorrect attachments and regulates the timing of mitotic progression via the spindle assembly checkpoint [8]. During anaphase, the CPC associates with the central spindle and during cytokinesis with the equatorial cortex and midbody to control cell abscission [16-20].

CPC localisation at centromeres has been suggested to depend on the co-existence of two histone modifications: Haspin-mediated phosphorylation on histone H3 Thr3 (H3T3ph) and Bub1-mediated phosphorylation on histone H2A Thr120 (H2AT120ph). According to the proposed models, H3T3ph is directly recognised by the BIR domain of Survivin [1-3] and H2AT120ph is read by hSgo1, which then recruits Borealin [1, 21]. However, Haspin depletion by siRNA does not abolish CPC association to chromatin [3] and Survivin is not sufficient to achieve centromeric enrichment or chromosomal association of CPC in cells expressing Borealin lacking its C-terminal half [10]. These observations collectively highlight a central question that remains unanswered. How does the CPC associate with chromosomes during early prophase prior to its H3T3Ph-mediated centromeric enrichment?

\section{Results and Discussion}

\section{Borealin nucleosome binding is essential for chromosome association of the CPC}

Consistent with our previous observations (Jeyaprakash et al., 2007), transient expression of a Myc-tagged Borealin lacking the first 10 aa and C-terminal half (Borealin 10-109) failed to 
rescue the siRNA-mediated depletion of endogenous Borealin and the CPC was completely excluded from chromosomes during the early stages of mitosis leading to chromosome congression defects (Figure 1A, B and S1A, B). This led us to hypothesise a direct role for Borealin in mediating CPC-chromosome interactions. To test this, we reconstituted nucleosome core particles (NCPs) containing homogenous H3T3ph modification in vitro and performed Electrophoretic Mobility Shift Assays (EMSAs) with recombinant CPC localisation module (CPC_LM: Borealin-Survivin-INCENP1-58, Figure S1C). The CPC_LM showed clear binding to modified NCPs as evidenced by its retarded mobility (Figure 1C). Interestingly,

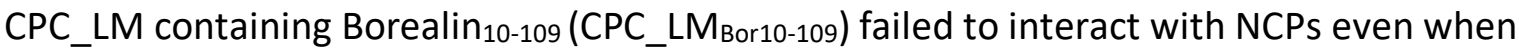
mixed at a 32 times molar excess (Figure $1 C$ ). This is particularly surprising, as several studies have shown previously that Survivin can bind synthetic N-terminal HH3 peptides phosphorylated at Thr3 through its BIR domain [1-3]. To test if Survivin on its own could bind H3T3ph tail in the context of NCPs, we analysed the binding of purified Survivin and H3T3ph NCPs by EMSA. Strikingly, Survivin did not bind H3T3ph NCPs (Figure 1D) possibly due to a lack of H3 tail accessibility within the NCP. Together, these data demonstrate Borealin to be a major contributor to CPC-nucleosome interactions. Considering Borealin's direct role, we next asked if the CPC_LM can bind unmodified NCPs. Interestingly, the CPC retarded the mobility of unmodified NCPs in the EMSA assays confirming its binding to unmodified NCPs (Figure 1E). These observations establish that the CPC can bind NCPs in a H3T3ph-independent manner and the interaction is mainly mediated by Borealin.

As $\mathrm{H} 3 \mathrm{~T} 3 \mathrm{ph}$ has been proposed to be critical for concentrating the CPC at inner-centromeres, we speculated that phosphorylation on $\mathrm{H} 3 \mathrm{Thr} 3$ might positively influence nucleosome binding affinity of the CPC. To address this, we performed Surface Plasmon Resonance (SPR) experiments by flowing CPC at different concentrations over the sensor surface containing immobilised NCP and measured steady-state binding affinities. While the CPC_LM interacted with unmodified NCPs with a $\mathrm{K}_{d}$ of $295.2 \pm 40.9 \mathrm{nM}$, interaction with H3T3ph NCPs was 3-fold tighter at $102.8 \pm 34.2 \mathrm{nM}$ (Figure 1F). Interestingly this increase in affinity for phosphorylated NCPs was due to Survivin binding to the H3 N-terminal tail, as a CPC containing a Survivin BIR mutant deficient for binding the phosphorylated $\mathrm{H} 3$ tail (CPC_LMSUR MUT, Figure S1D), bound both modified and unmodified NCPs with a similar affinity (Figure 1F). Thus, our data show that although the Survivin-H3 interaction is not 
essential for CPC chromatin binding per se, it enhances the affinity of the CPC for modified NCPs. Consistent with EMSAs, SPR failed to detect any binding of Survivin on its own to NCPs even when $\mathrm{H} 3$ is phosphorylated at Thr3 (Figure 1F).

\section{$\mathrm{N}$-terminal 10 amino acids and C-terminal half of Borealin are required for CPC-chromatin interaction}

Considering the essential contribution of Borealin towards nucleosome binding, we next mapped the regions of Borealin directly involved in nucleosome interaction. We reconstituted several versions of CPC_LM complexes containing different Borealin mutants (designed based on its domain architecture) (Figure 1A and S2A) and tested them in EMSA assays with and without H3T3 phosphorylation on NCPs (Figure 2A and S2B). Deleting a well-conserved unstructured central region of Borealin (aa residues 110-206, CPC_LM BOR $_{\text {B }}$ $\Delta$ loop, Figure S2C) abolished CPC binding to NCP almost completely. The deletion of either the $\mathrm{N}$-terminal 10 (CPC_LMBOR 10-end) or C-terminal 59 aa of Borealin (CPC_LMBOR 10-221) also caused a noticeable reduction in binding (Figure 2A and S2B). Considering the qualitative nature of the EMSA assay, we evaluated the NCP-binding affinities of mutant CPC complexes in SPR assays (Figure 2B and S3A). Consistent with EMSA assays, CPC_LMBOR 10-end showed 3fold reduction in binding affinity as compared to CPC_LM. Both CPC_LM LOR $\triangle$ loop and CPC_LMBOR 10-221 exhibited even weaker NCP binding with measured affinities in the $\mu \mathrm{m}$ range (Figure $2 \mathrm{~B}$ ). Together our data show that the $\mathrm{N}$-terminal 10 aa and $\mathrm{C}$-terminal half of Borealin contribute to nucleosome binding, possibly through multiple physical contacts.

Having dissected the contribution of Borealin for nucleosome binding in vitro, we evaluated the behavior in vivo of Borealin mutants showing reduced NCP binding in siRNA rescue

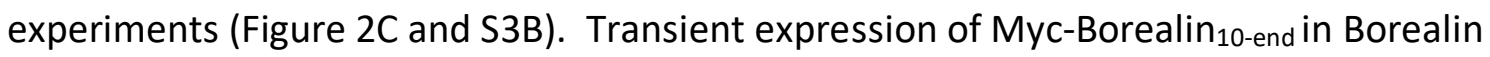
siRNA-depleted HeLa cells, resulted in a $50 \%$ reduction of the centromeric association of the CPC (Figure 2D and S3C). In contrast, expression of Myc-Borealin ${ }_{\triangle l o o p}$ or Borealin $10-221$ resulted in almost complete exclusion of the CPC from chromosomes. Collectively, these observations demonstrate that Borealin-mediated nucleosome binding is essential for chromosome association of the CPC in vivo. 


\section{CPC-Nucleosome binding is mediated by multivalent interactions predominantly involving}

\section{Borealin}

To gain structural insight into the underlying mechanism, the CPC-NCP complexes (Figure S4A) were crosslinked using EDC and analysed by mass spectrometry (Figure S4B, C).

Consistent with our in vitro binding studies, regions of Borealin shown here to be critical for nucleosome binding made extensive contacts with NCPs, whereas Survivin interactions are mostly limited to the BIR domain and the $\mathrm{H} 3 \mathrm{~N}$-terminal tail (Figure 3A and S4D) as expected. Mapping the crosslinks onto the three dimensional structures of NCP and CPC (Figure 3B) suggests a model where the localisation module of the CPC together with a highly basic $\mathrm{N}$-terminal tail of Borealin docks onto the acidic patch formed between $\mathrm{H} 2 \mathrm{~A}$ and H2B, a surface commonly involved in nucleosome recognition [22-26]. This interaction may orient the Survivin BIR domain to facilitate binding of Histone $\mathrm{H} 3$ tail phosphorylated at Thr3. Notably, Borealin loop residues trace along the DNA-histone interface implying its major contribution to nucleosome binding involves both protein-protein and possibly protein-DNA contacts.

\section{Borealin-mediated chromosome association of the CPC is an upstream requirement for its} Haspin and Bub1-mediated centromeric enrichment

As Haspin activity has been suggested to be stimulated by the Aurora B kinase, we next evaluated the impact of Borealin-mediated chromosome association of CPC on Haspin activity. Strikingly, in Borealin depleted cells, H3T3 phosphorylation was reduced to low levels (Figure 4A). While the expression of Myc-Borealin rescued these H3T3ph levels, expression of a Borealin mutant incapable of chromosome association (Myc-Borealin ${ }_{\Delta l o o p}$ ) failed to do so. Likewise, Borealin depletion led to a decrease in the levels of H2AT120 phosphorylation (Figure 4B), which could be rescued with Borealin, but only partially with Borealin ${ }_{\Delta l o o p}$. Notably, Haspin depletion, which resulted in reduced H3T3 phosphorylation and as a consequence diffused CPC localization along the chromosome arms, did not affect H2AT120 phosphorylation (Figure S5A). Together, our data demonstrates that CPC binding to nucleosomes is an upstream requirement for Haspin and Bub1 activities and for Haspin/Bub1-mediated CPC enrichment at centromeres. 
In summary, our data suggest a mechanism for the chromosome association of CPC essential for error-free chromosome segregation (Figure 4C). During early mitosis when there is little or no H3T3ph [27] the CPC binds chromatin in a histone modificationindependent manner, mainly via Borealin interactions involving multiple contacts with the histone octamer and possibly also with the DNA. Chromatin association of the CPC activates Haspin and Bub1, which in turn phosphorylate H3 Thr3 and H2A Thr120, respectively. These histone phosphorylation marks increase the affinity of CPC for chromatin due to Survivin BIR interaction with H3T3ph [1-3] and possibly binding of Borealin to Sgo1 [1, 21]. This facilitates CPC enrichment at inner centromeres during prometaphase and metaphase. In the future, it will be important to understand how such extensive multivalent interactions between CPC and chromatin are weakened to transfer the CPC from chromatin to the central spindle during late metaphase/anaphase $[28,29]$.

\section{Acknowledgements}

We thank the staff of the Edinburgh Protein Production Facility and the Centre Optical Instrumentation Laboratory for their help. We also thank Tony Ly, Dhanya Cheerambathur and Andrew Goryachev for critical reading of the manuscript. The Wellcome Trust generously supported this work through a Senior Research Fellowship (202811) to AAJ, a Principal Research Fellowship to WCE (073915), a Senior Research Fellowship to JR (084229), a Sir Henry Dale Fellowship to PV (104175/Z/14/Z), a Centre Core Grant (092076 and 203149) and an instrument grant (091020) to the Wellcome Trust Centre for Cell Biology, a Multi-User Equipment grant 101527/Z/13/Z to the EPPF. JGR was supported by the Marie Curie Action PloidyNet, funded by the European Union Seventh Framework Programme (FP7/2007-2013) under Grant Agreement Number 607722. 
A

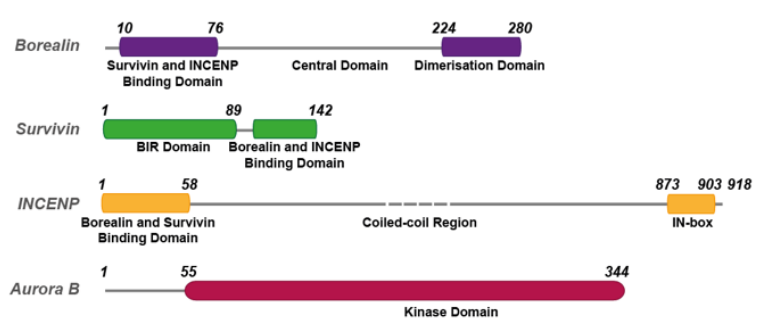

B

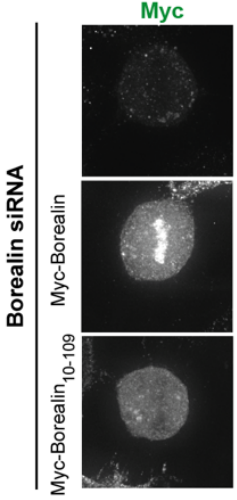

Survivin

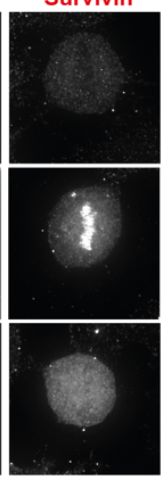

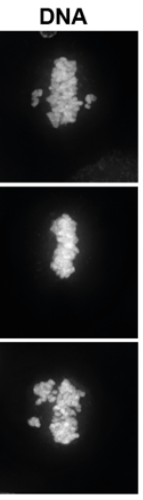

Merge

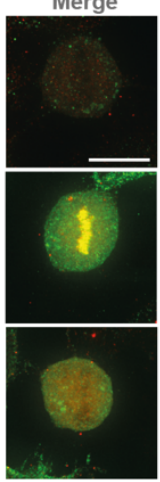

C

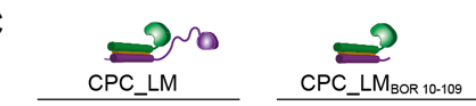

$0 \longdiv { 2 0 4 0 8 0 1 6 0 3 2 0 6 4 0 } \div \overline { 2 0 4 0 8 0 1 6 0 3 2 0 6 4 0 } \mathrm { nM }$

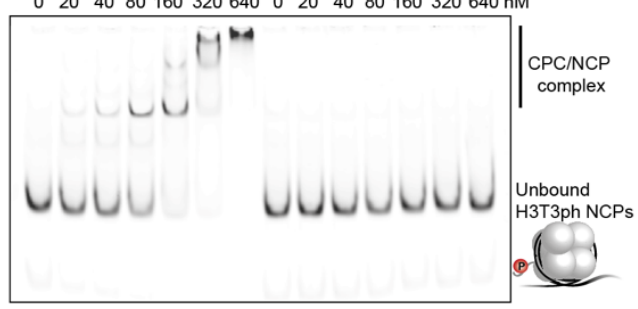

$\mathbf{F}$
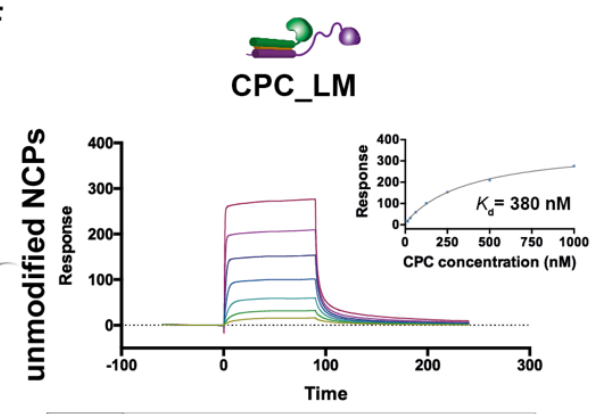

\begin{tabular}{l|l}
$K_{d}$ & $295.2 \pm 40.9 \mathrm{nM}$
\end{tabular}

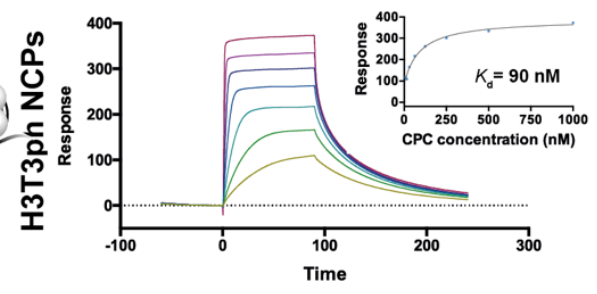

$$
\begin{array}{|l|l|}
\hline K_{d} & 102.8 \pm 34.2 \mathrm{nM} \\
\hline
\end{array}
$$

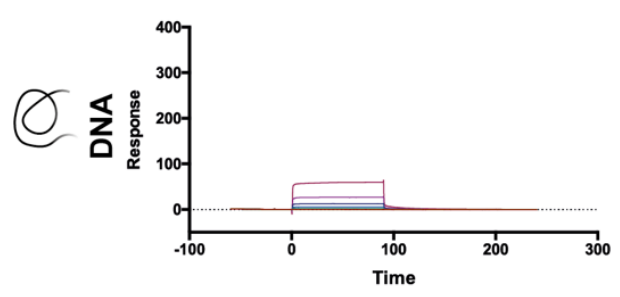

D

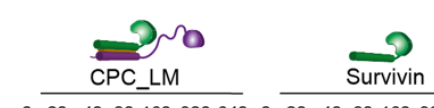

$0 \longdiv { 2 0 4 0 8 0 1 6 0 3 2 0 6 4 0 } 0 \overline { 2 0 4 0 8 0 1 6 0 3 2 0 6 4 0 \mathrm { nM } }$

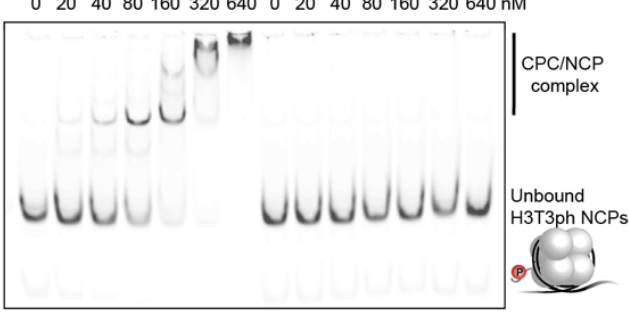

E

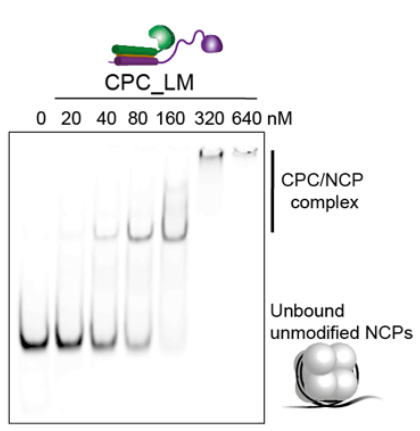

$2 \sim 0$

CPC_LM

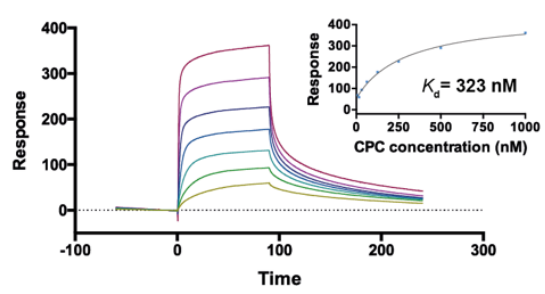

\begin{tabular}{|l|l|}
\hline$K_{d}$ & $309 \pm 14 \mathrm{nM}$ \\
\hline
\end{tabular}
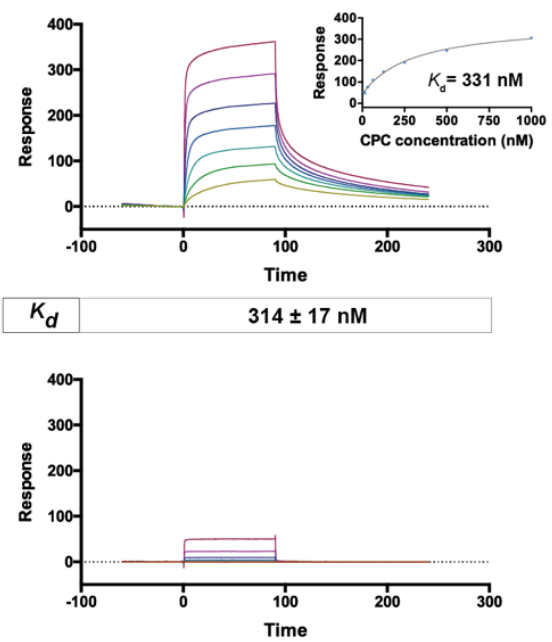
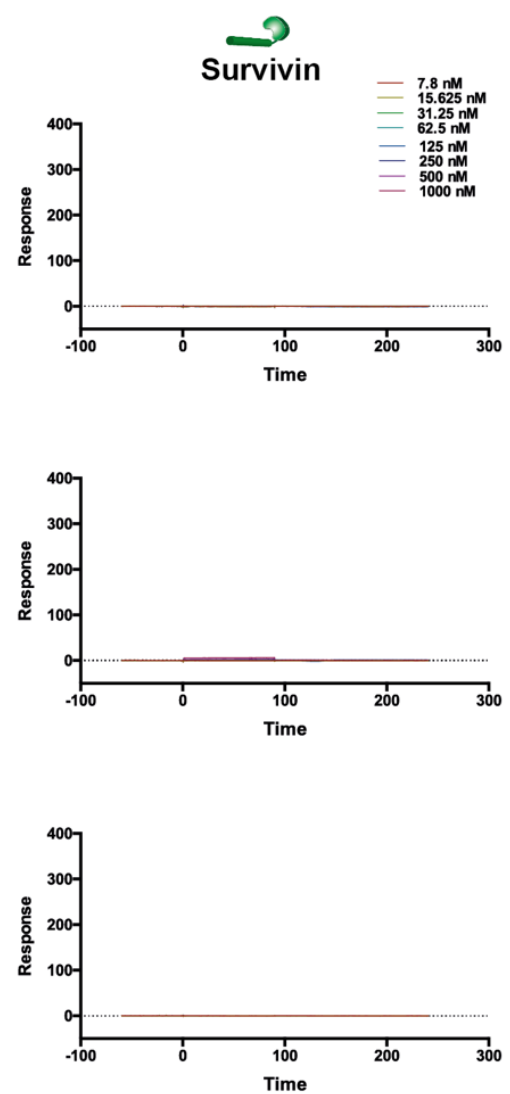

Figure 1 Borealin nucleosome binding is essential for chromosome association of the CPC.

(A) Domain architecture of the subunits of the Chromosomal Passenger Complex (CPC). (B)

Representative fluorescence images of a siRNA rescue assay for Borealin $10-109$ fragment. Immunofluorescent staining of Myc and Survivin in HeLa cells co-transfected with siRNA 
duplexes targeting the 3'UTR region of Borealin and Myc-Borealin constructs. Hoechst was used for DNA staining. Scale bar, $10 \mu \mathrm{m}$. All cells transfected with the siRNA and MycBorealin $_{10-109}$ fragment showed exclusion of the CPC complex from the chromatin. (C,E) Native PAGE analysis of EMSA assays performed with increasing concentrations of recombinant CPC localisation module (CPC_LM) containing different Borealin fragments and either 20 nM phosphorylated (H3T3ph) (C) or unmodified IR700-labelled NCPs (E). (D) EMSA assays performed with increasing concentrations of Survivin with $20 \mathrm{nM}$ IR700-labelled H3T3ph NCPs. (F) Representative SPR sensorgrams of the interaction between different CPC_LM complexes (CPC_LM, CPC_LMsUR MUT) or Survivin and unmodified (top) or H3T3ph NPCs (middle) or DNA (bottom) immobilized on the surface of a neutravidin sensor chip. Mean values $(n \geq 2, \pm S E M)$ determined for the equilibrium dissociation constant $\left(K_{d}\right)$ are shown in boxes underneath the sensorgrams. 
A
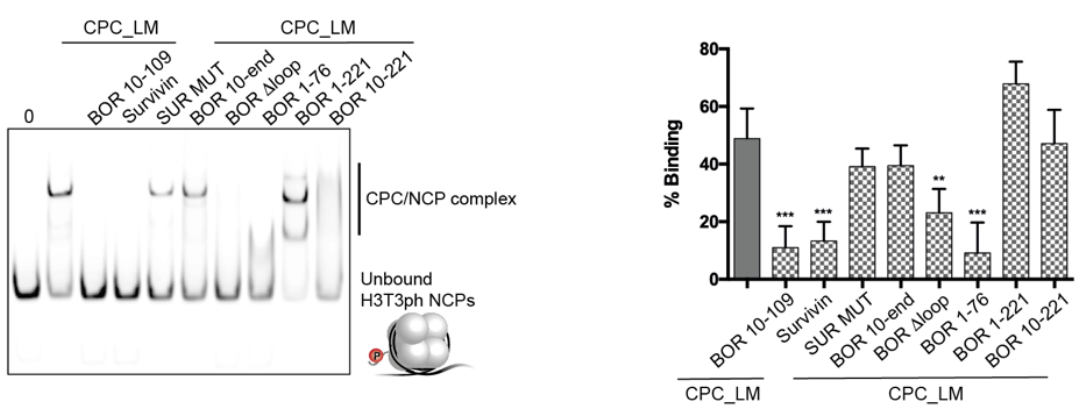

B
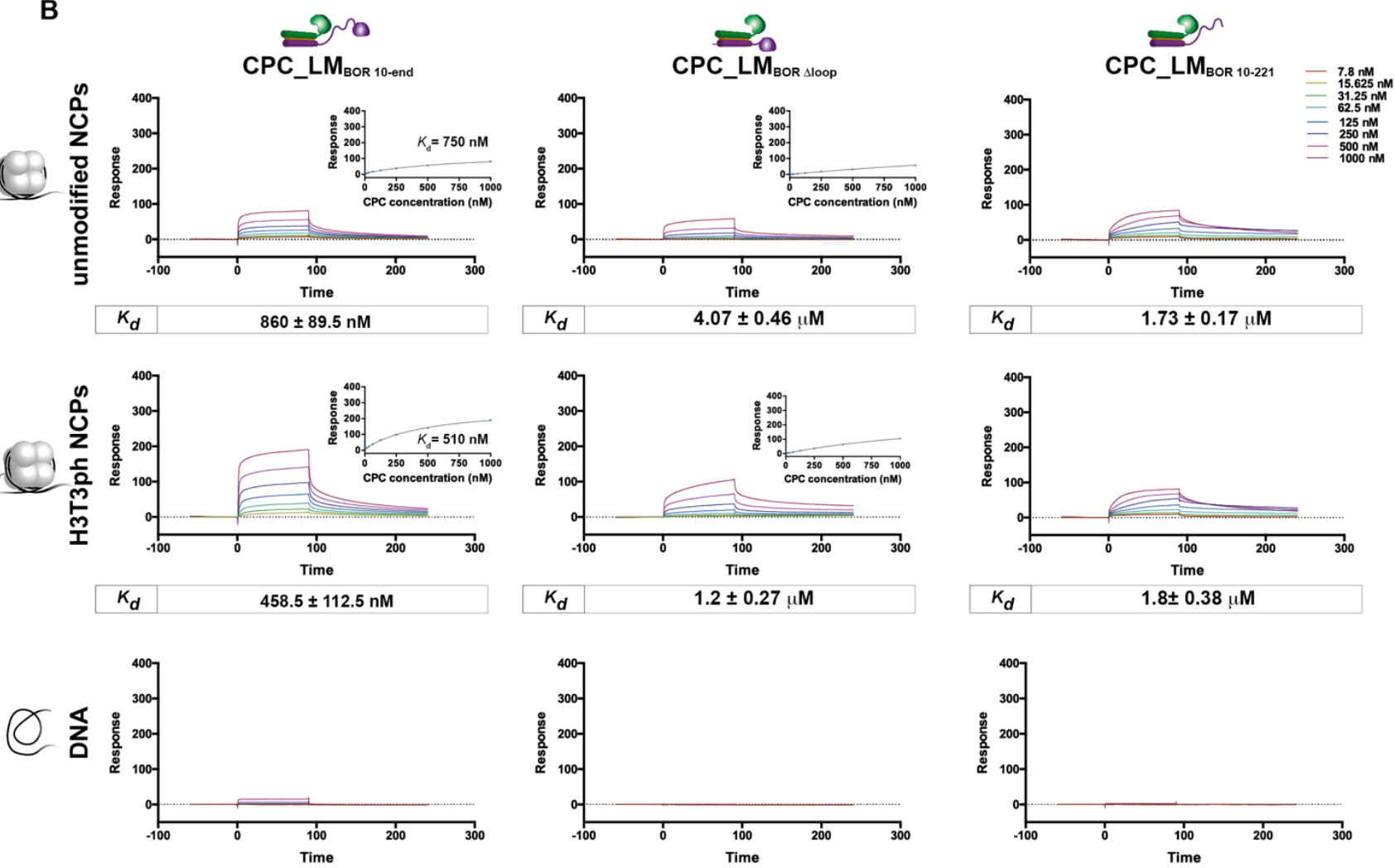

C

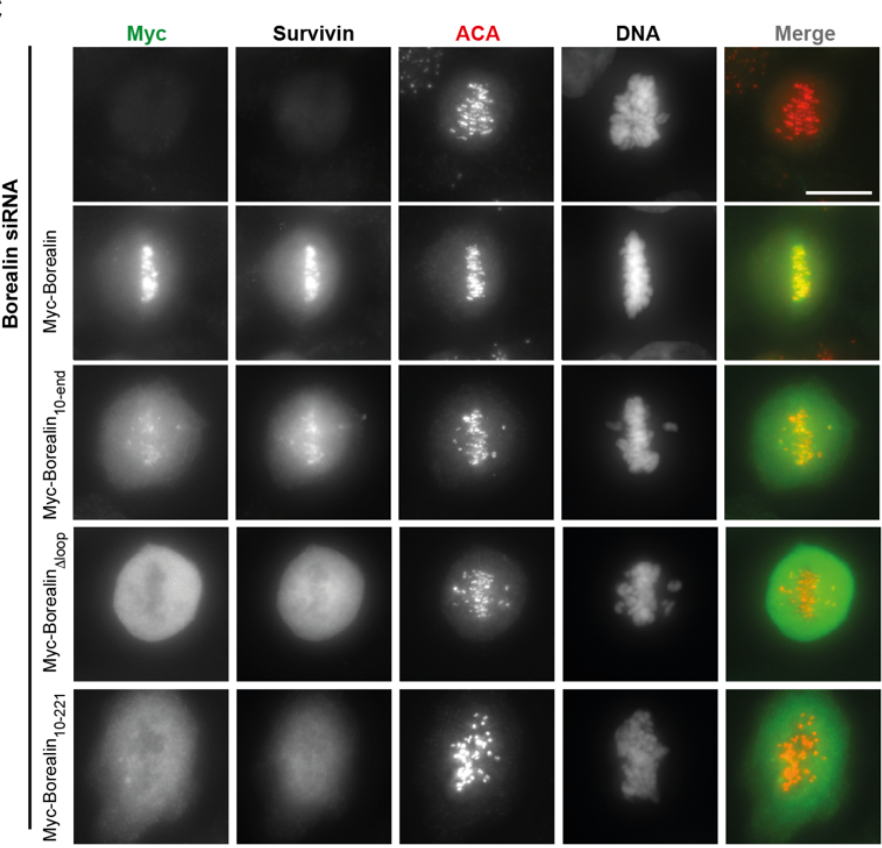

D

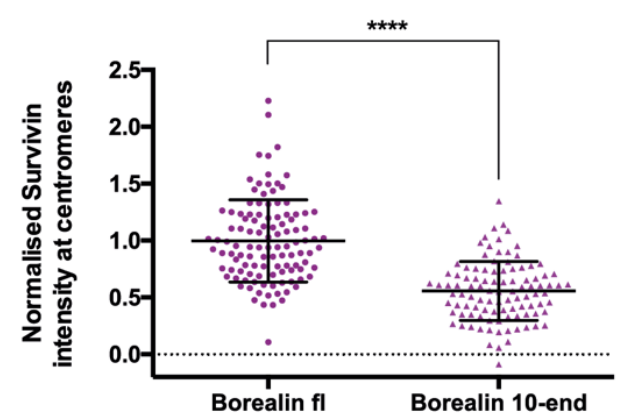

Figure $\mathbf{2} \mathrm{N}$-terminal 10 amino acids and C-terminal half of Borealin are required for CPC-

chromatin interaction. 
(A) Native PAGE analysis of EMSA assays performed with recombinant CPC_LM containing different Borealin truncations binding to IR700-labelled H3T3ph NCPs (left) and quantification of binding (right). Concentrations of the NCP and the CPC used in the assay were $20 \mathrm{nM}$ and $160 \mathrm{nM}$, respectively. Mean of \% of binding \pm standard deviation (SD); $n=5$; $* * \mathrm{P} \leq 0.01, * * * \mathrm{P} \leq 0.001$, unpaired $t$-test. (B) Representative SPR sensorgrams of the interaction between different CPC_LM complexes (CPC_LMBOR 10-end, CPC_LM CPC_LMBOR 10-221) and unmodified (top) or H3T3ph NCPs (middle) or DNA (bottom). Mean values ( $n \geq 3, \pm$ SEM) determined for the equilibrium dissociation constant $\left(K_{d}\right)$ are shown in boxes underneath the sensorgrams. (C) Representative fluorescence images of a rescue assay for Borealin, Borealin ${ }_{10-e n d}, B$ Borealin ${ }_{\Delta l o o p}$, Borealin ${ }_{10-221}$ constructs. Immunofluorescent staining of Myc, Survivin and ACA in HeLa cells co-transfected with siRNA duplexes targeting the 3'UTR region of Borealin and Myc-Borealin constructs. Hoechst was used for DNA staining. Scale bar, $10 \mu \mathrm{m}$. (D) Quantification of Survivin levels at the centromeres for the siRNA-rescue assays with Myc-Borealin ( $n=111$ cells) and Myc-Borealin $10-e n d$ ( $n=101$ cells) shown in (C) (three independent experiments, mean \pm SEM, Mann-Whitney test; $P<0.0001$ ). For constructs Myc-Borealin ${ }_{\Delta l o o p}$ and Myc-Borealin $10-221$, all metaphase cells exhibited total exclusion of the CPC complex from the chromatin. 
A

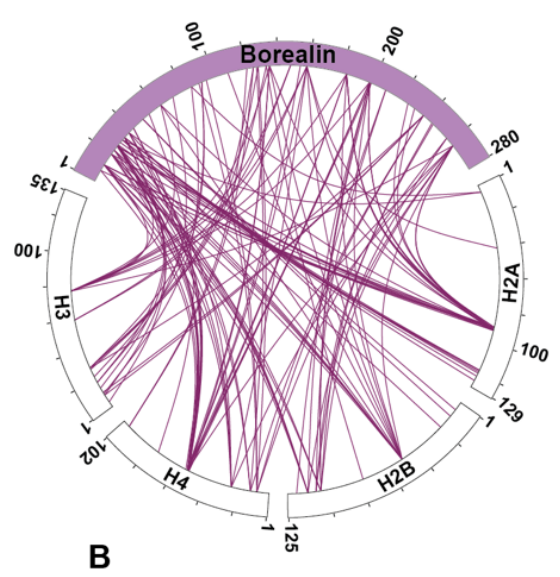

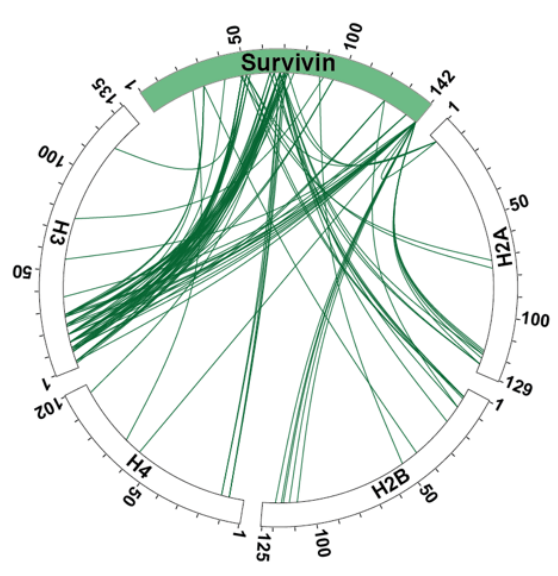

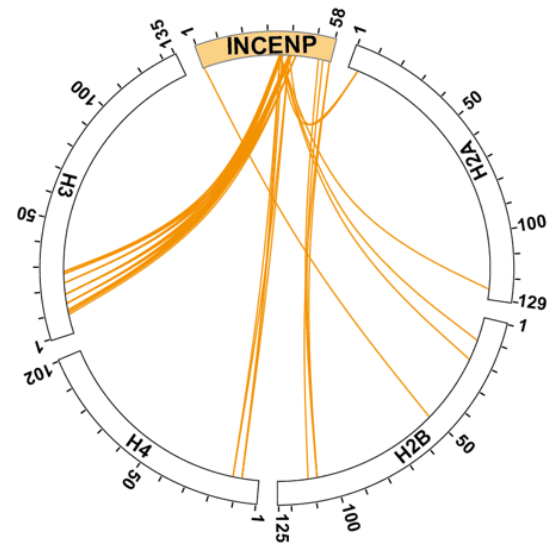

CPC Localisation Module

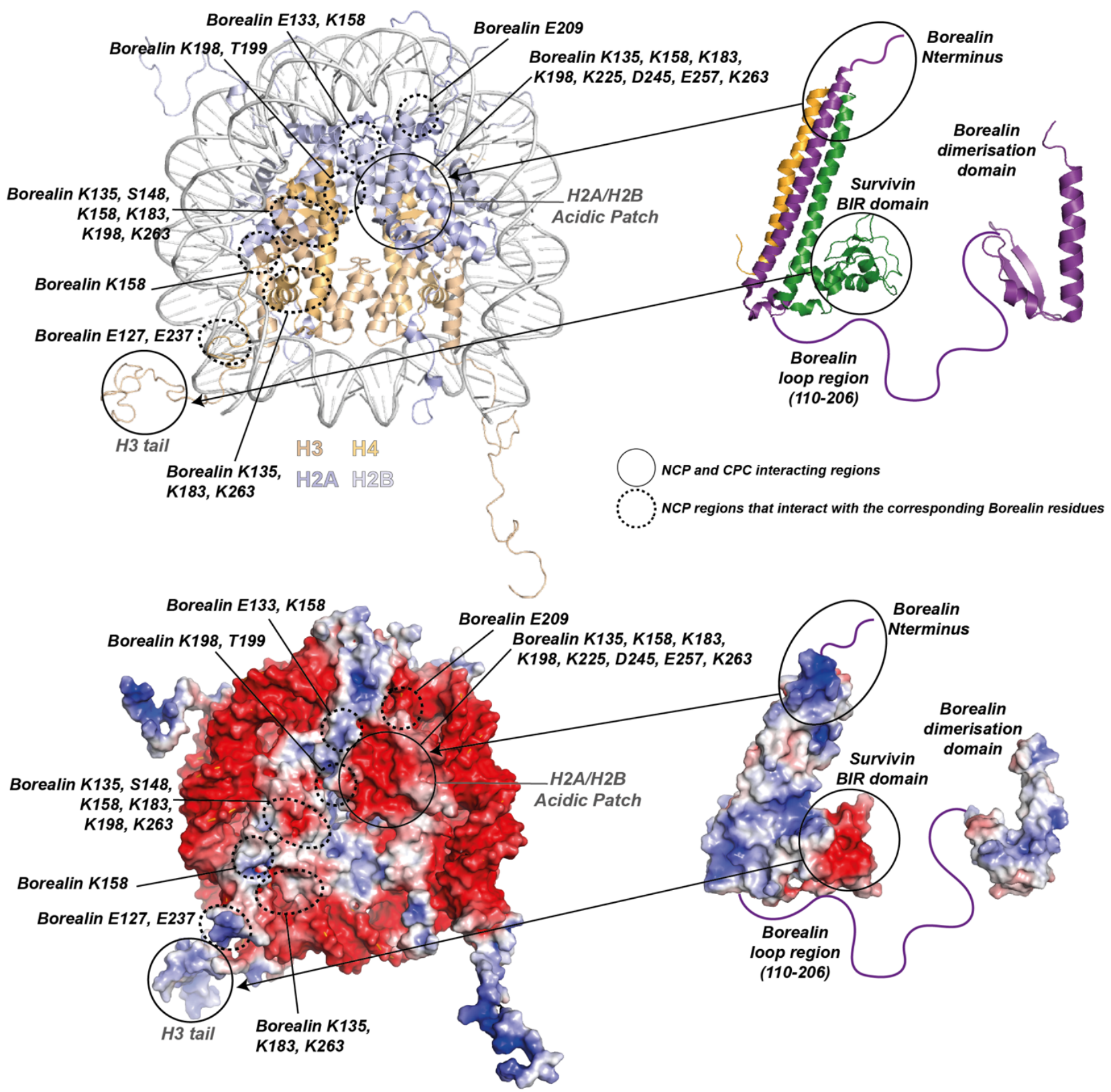

Figure 3 CPC-Nucleosome binding is mediated by multivalent interactions predominantly involving Borealin. 
(A) Circle view of the crosslinks between subunits of the CPC (Borealin-purple, Survivingreen, INCENP-yellow) and histones from unmodified NCPs. Intermolecular contacts involving Borealin, Survivin and INCENP and histones are shown as purple, green or yellow lines, respectively, using XiNET (Ref: PMID: 25648531). (B) Cartoon representation of the crystal/NMR structures of the NCP (PDB: 1kx5, [30]) and CPC (CPC core PDB: 2qfa, [10] and Borealin Dimerisation domain PDB: 2kdd, [31]) (top). Surface representation of the NCP and the CPC coloured based on the electrostatic surface potential calculated using APBS in Pymol v2.0.6 (bottom). 
A

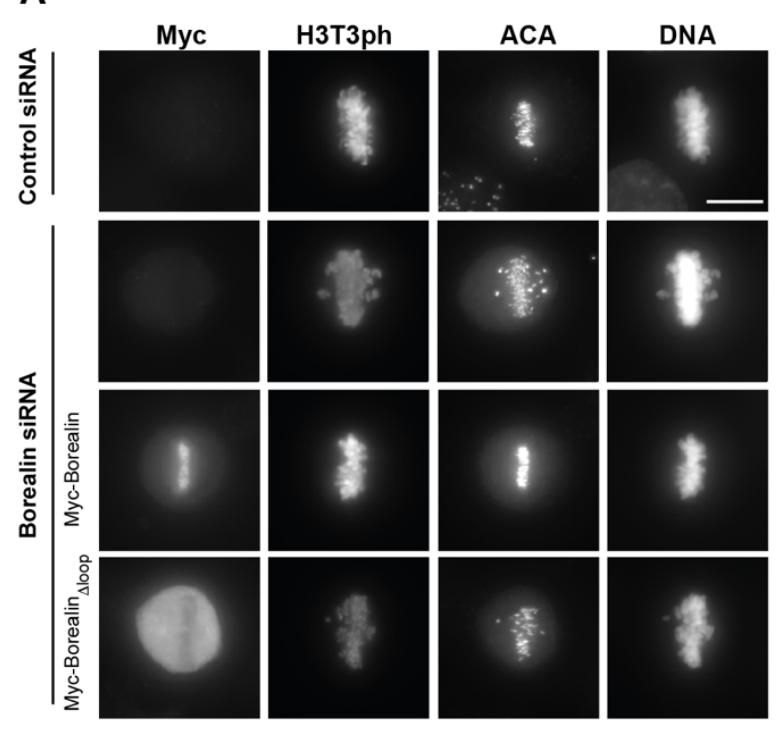

B

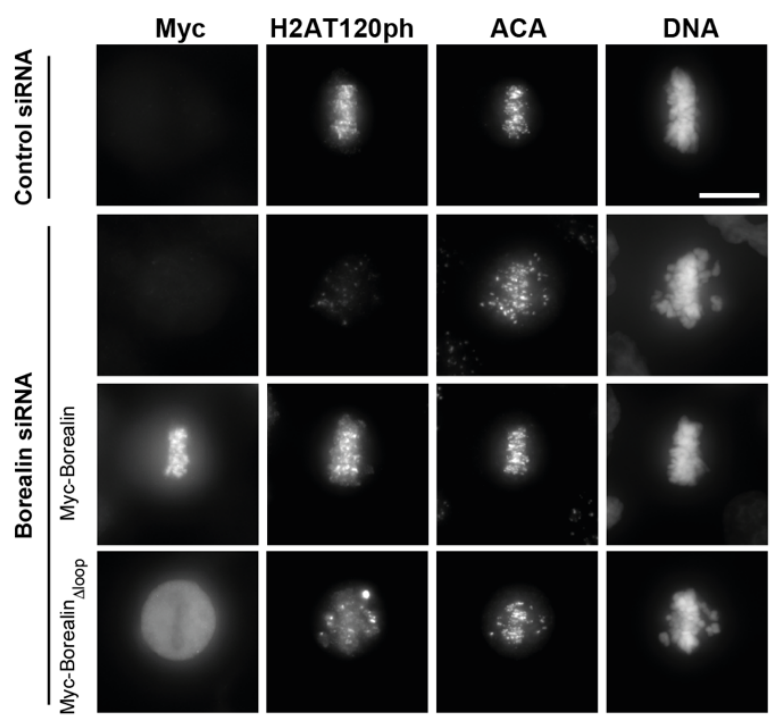

C

Prophase/Prometaphase

Metaphase

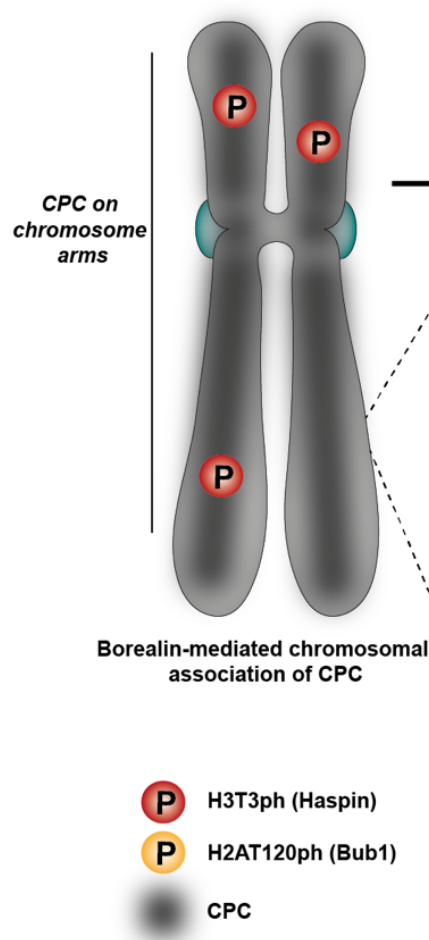

Figure 4 Borealin-mediated chromosome association of the CPC is an upstream requirement for its Haspin and Bub1-mediated centromeric enrichment.

(A, B) Immunofluorescence analysis of ACA and H3T3ph (A) or H2AT120ph (B) levels upon

Borealin depletion using siRNA duplexes and rescue with different Borealin constructs.

Hoechst was used for DNA staining. Scale bar, $10 \mu \mathrm{m}$. (C) Model for Borealin-mediated chromatin association of the CPC and subsequent centromere enrichment. 


\section{STAR Methods}

\section{Protein Expression and purification of the CPC}

Survivin was cloned as a 3C-cleavable His-GFP tagged protein in a pRSET vector (Thermo Fisher Scientific). The different Borealin fragments were cloned as a TEV-cleavable Histagged protein in a pETM vector (gift from C. Romier, IGBMC, Strasbourg), and INCENP 1-58 was cloned as an untagged protein in a pMCNcs vector.

The CPC subunits were co-expressed in BL21(DE) pLysS strain. Cells were lysed in lysis buffer (25 mM Hepes pH 7.5, $500 \mathrm{mM} \mathrm{NaCl}, 25 \mathrm{mM}$ Imidazole, $2 \mathrm{mM} \beta$-mercaptoethanol) and purified using a $5 \mathrm{ml} \mathrm{HisTrap} \mathrm{HP} \mathrm{column} \mathrm{(GE} \mathrm{Healthcare).} \mathrm{The} \mathrm{protein-bound} \mathrm{column} \mathrm{was}$ washed with lysis buffer, followed by wash buffer $(25 \mathrm{mM}$ Hepes pH 7.5, $1 \mathrm{M} \mathrm{NaCl}, 50 \mathrm{mM}$ $\mathrm{KCl}, 10 \mathrm{mM} \mathrm{MgCl}, 25 \mathrm{mM}$ Imidazole, $2 \mathrm{mM} \mathrm{ATP}, 2 \mathrm{mM} \beta$-mercaptoethanol). Elution buffer (25 mM Hepes pH 7.5, 500 mM NaCl, 500 mM Imidazole, 2 mM $\beta$-mercaptoethanol) was used to elute the proteins. Tags were cleaved overnight with $3 \mathrm{C}$ and TEV proteases while dialysing against $25 \mathrm{mM}$ Hepes $\mathrm{pH} 7.5,150 \mathrm{mM} \mathrm{NaCl}, 4 \mathrm{mM}$ dithiothreitol (DTT) at $4^{\circ} \mathrm{C}$. The complex was further purified by a cation exchange chromatography (HiTrap SP, GE Healthcare) followed by gel filtration using a Superdex 200 increase 10/300 column (GE Healthcare) pre-equilibrated with $25 \mathrm{mM}$ Hepes pH 8, $200 \mathrm{mM} \mathrm{NaCl}, 4 \mathrm{mM}$ DTT.

\section{Expression and purification of recombinant histones and refolding of histone octamers}

Human $\mathrm{H} 2 \mathrm{~A}$ and $\mathrm{H} 2 \mathrm{~B}$ and Xenopus laevis $\mathrm{H} 3$ and $\mathrm{H} 4$ were purified as described before [32]. Briefly, H2A, H2B and H3 were expressed in BL21 (DE3) pLysS cells while H4 were expressed in BL21 cells using LB media. The histones were purified from inclusion bodies using a Dounce glass/glass homogenizer. After solubilisation of the inclusion bodies we performed a the three-step dialysis against urea dialysis buffer $(7 \mathrm{M}$ Urea, $100 \mathrm{mM} \mathrm{NaCl}, 10 \mathrm{mM}$ Tris $\mathrm{pH}$ 8, $1 \mathrm{mM}$ EDTA, $5 \mathrm{mM} \beta$-mercaptoethanol). The sample was then applied to a HiTrap Q anion exchange column and a HiTrap SP cation exchange column (GE Healthcare). Histones were eluted from the HiTrap SP column using a linear gradient from $100 \mathrm{mM}$ to $1 \mathrm{M} \mathrm{NaCl}$ in $7 \mathrm{M}$ Urea, $10 \mathrm{mM}$ Tris $\mathrm{pH}$ 8, $1 \mathrm{mM}$ EDTA and $1 \mathrm{mM}$ DTT. Purified recombinant histones were dialysed against water containing $5 \mathrm{mM} \beta$-mercaptoethanol before lyophilization and storage at $-80^{\circ} \mathrm{C}$.

To generate histone $\mathrm{H} 3$ phosphorylated at threonine 3 by native chemical ligation, histone H3 lacking residues 1-31 containing a threonine-to-cysteine substitution at position 32 and 
a cysteine-to-alanine substitution at position 110 (H3A1-31 MT32C C110A) was expressed and purified as described above. Native chemical ligation reactions with $\mathrm{H} 3 \Delta 1-31 \mathrm{MT} 32 \mathrm{C}$ C110A and the N-terminal H3 peptide ARTPhKQTARKSTGGKAPRKQLATKAARKSAPA containing a C-terminal benzyl thioester (Peptide Protein Research Ltd., Fareham, UK) were carried out in $6 \mathrm{M}$ Guanidine $\mathrm{HCl}, 250 \mathrm{mM}$ sodium phosphate buffer $\mathrm{pH} 7.2,150 \mathrm{mM}$ 4mercaptophenylacetic acid (MPAA), $50 \mathrm{mM}$ TCEP for $72 \mathrm{~h}$ at room temperature with constant agitation. Reactions were dialysed three times against $7 \mathrm{M}$ urea, $100 \mathrm{mM} \mathrm{NaCl}$, $10 \mathrm{mM}$ Tris pH 8, 1 mM EDTA, 1 mM DTT. Ligated full-length H3T3Ph histone was separated from unligated truncated histone through cation exchange chromatography on a monoS column (GE) and then dialysed against water containing $5 \mathrm{mM} \beta$-mercaptoethanol before lyophilization and storage at $-80^{\circ} \mathrm{C}$.

Histone octamers were obtained as previously described [32]. Briefly, lyophilized histones were resuspended in unfolding buffer (7M Guanidine $\mathrm{HCl}, 20 \mathrm{mM}$ Tris $\mathrm{pH}$ 7.5, $10 \mathrm{mM}$ DTT) and mixed to equimolar ratios. The histone mix was then dialysed three times against $500 \mathrm{ml}$ of refolding buffer (10 mM Tris pH 8, 2M NaCl, 1 mM EDTA, $5 \mathrm{mM} \beta$-mercaptoethanol). The octamers were obtained by running the histone mix on a size exclusion chromatography column (Superdex 200 increase 10/300, GE Healthcare) pre-equilibrated with refolding buffer and stored at $-80^{\circ} \mathrm{C}$.

\section{Nucleosome core particle reconstitution}

A pBS-601 Widom vector was used to amplify the 147bp 601 Widom positioning sequence with unlabeled, 5' IR700- or biotin-labelled primers. Mononucleosomes were obtained by using the salt gradient dialysis method [32]. After optimization of the octamer-DNA ratio, the histone octamer-DNA mix was dialysed against TE buffer (10 mM Tris pH 8, 1 mM EDTA, $50 \mathrm{mM} \mathrm{NaCl}$ ) overnight by gradually decreasing the ionic strength from $2 \mathrm{M}$ using a peristaltic pump.

\section{Electrophoretic Mobility Shift Assay}

Different concentrations of recombinant CPC were added to $20 \mathrm{nM}$ IR700-labelled NCPs in reaction buffer (10 mM Tris pH 7.5, $100 \mathrm{mM} \mathrm{NaCl}, 1 \mathrm{mM} \mathrm{MgCl}_{2}, 1 \mathrm{mM}$ DTT, 1\% Glycerol, $0.1 \mathrm{mg} / \mathrm{ml} \mathrm{BSA}$ ). Reactions were incubated $1 \mathrm{~h}$ at $4{ }^{\circ} \mathrm{C}$ and run in a $6 \%$ polyacrylamide native gel in $0.5 \mathrm{X}$ TBE buffer at $100 \mathrm{~V}$ for $2 \mathrm{~h}$ at $4^{\circ} \mathrm{C}$. The fluorescent bound and unbound NCPs were 
detected with Odyssey CLx Infrared Imaging System (LI-COR Biosciences). The fluorescent signal of the band corresponding to unbound NCPs was quantified using Image J. Values were plotted and statistically analysed using Prism 6.0 (GraphPad Software, Inc).

\section{Surface Plasmon Resonance}

Surface plasmon resonance experiments were performed using a BIAcore T200 instrument (GE Healthcare). Strepavidin coated sensor surfaces (Sensor Chip SA; GE Healthcare) were primed prior to ligand immobilization by three sequential 30 second-injections of $50 \mathrm{mM}$ $\mathrm{NaOH} ; 1 \mathrm{M} \mathrm{NaCl}$ followed by 180 seconds of Running Buffer (25mM Hepes, $250 \mathrm{mM} \mathrm{NaCl} 1$ $\mathrm{mM}$ DTT and 0.05\% Tween-20, pH8) at $30 \mu \mathrm{l} / \mathrm{min}$. Biotinylated ligands (in vitro reconstituted NCPs and DNA) were then immobilized on respective flow-cells by injecting $20 \mathrm{nM}$ solutions of respective ligands at $5 \mu \mathrm{l} / \mathrm{min}$, and varying the contact time until the Response Units (RU) on the surface reached $~ 500 \mathrm{RU}$. Immediately prior to each SPR experiment, samples (CPC complexes, NCPs and DNA) were dialysed against running buffer for $1 \mathrm{~h}$ at $4{ }^{\circ} \mathrm{C}$. Two-fold dilution series, of respective analytes, from $7.8 \mathrm{nM}-1 \mu \mathrm{M}$ in Running Buffer, were injected over the sensor surface at $100 \mu \mathrm{l} / \mathrm{min}$ at $8{ }^{\circ} \mathrm{C}$, with 90 second association and 150 second dissociation times. This was then immediately followed by a 300 second regeneration phase in Running Buffer. A neutravidin surface without ligand served as a reference flow-cell for bulk correction. Apparent equilibrium dissociation constants were calculated from the sensorgrams by global fitting of a steady state, 1:1 binding model, with mass transport considerations, using the analysis software (v2.02), provided with the BIAcore T200 instrument. Data was replotted for clarity using Prism 6.0 (GraphPad Software Inc).

\section{Rescue experiments and Immunofluorescence Microscopy}

Depletion of endogenous Borealin using RNAi and rescue experiments were performed as previously described [9] using jetPRIME (Polyplus Transfection). HeLa cells were grown on coverslips in twelve-well plates and medium was changed $12 \mathrm{~h}$ after transfection. Cells were fixed in $4 \%$ paraformaldehyde (PFA) $36 \mathrm{~h}$ after transfection. The oligonucleotides targeting the 3' UTR of Borealin (5'-AGGTAGAGCTGTCTGTTCAdTdT-3') [9] or targeting luciferase as a control (5'-CGUACGCGGAAUACUUCGAdTdT-3') [33], were described previously. 
For quantification of the Survivin signal, HeLa CDK1 analogue sensitive (CDK1-as) cells were used [27]. Cells were synchronised for $14 \mathrm{~h}$ using $10 \mu \mathrm{M} 1 \mathrm{NM}-\mathrm{PP} 1$ and fixed $90 \mathrm{~min}$ after washout to increase the number of cells in metaphase.

RNAi depletion of Haspin was performed using oligonucleotides described previously [34] (siRNA ID 1093). Cells were transfected using jetPRIME and fixed in $4 \%$ PFA $48 \mathrm{~h}$ after transfection.

Following antibodies were used for indirect immunofluorescence: Anti-myc (1:200; 9E10; Merck Millipore), anti-Borealin (1:500; 147-3; MBL), anti-Survivin (1:500; NB500-201; Novus), anti-H3T3ph (1:500; 07-424; Upstate), anti-H2AT120ph (1:500, 61195, Active Motif), anti-ACA (1:300; 15-235; Antibodies Inc). Hoechst 33342 was used for DNA staining. Imaging was performed using a wide-field DeltaVision Spectris (Applied Precision) microscope with a 100x NA 1.4 PlanApo objective. Shown images are maximum intensity projections. The acquired images were deconvolved using SoftWoRx 3.6 (Applied Precision) and the centromere intensity of Survivin was quantified using an ImageJ plugin (DOI:

10.5281/zenodo.2574963). Briefly, the plugin quantifies the mean fluorescence signal of Survivin in a 2 pixel-wide ring immediately outside the centromere, defined with the ACA staining. For background subtraction, a selected area within the cytoplasm signal was selected. Statistical significance of the difference between normalised intensities at the centromere region was established by a Mann-Whitney test using Prism 6.0 (GraphPad Software).

\section{Chemical Crosslinking and MS analysis}

Crosslinking experiments of the CPC-NCP complexes were performed using 1-ethyl-3-(3dimethylaminopropyl) carbodiimide (EDC, Thermo Fisher Scientific) in the presence of Nhydroxysulphosuccinimide (NHS, Thermo Fisher Scientific). EDC is a zero-length chemical crosslinker capable of covalently linking primary amines of Lysine and the protein $\mathrm{N}$ terminus and to a lesser extend also hydroxyl groups of Serine, Threonine and Tyrosine with carboxyl groups of Aspartate/Glutamate. $8 \mu \mathrm{g}$ of CPC or CPC-NCP complexes were incubated with $30 \mu \mathrm{g}$ EDC and $66 \mu \mathrm{g}$ of N-hydroxysulphosuccinimide for 2 hours at room temperature. The crosslinking was stopped by the addition of $100 \mathrm{mM}$ Tris-Cl. Crosslinking products were resolved using 4-12 \% Bis-Tris NuPAGE (Invitrogen) for 5 min and briefly stained using Instant Blue (Expedeon). Bands were excised, and the proteins were reduced with $10 \mathrm{mM}$ 
DTT for $30 \mathrm{~min}$ at room temperature, alkylated with $55 \mathrm{mM}$ iodoacetamide for $20 \mathrm{~min}$ at room temperature and digested using $13 \mathrm{ng} / \mu \mathrm{l}$ trypsin (Promega) overnight at $37^{\circ} \mathrm{C}$. The digested peptides were loaded onto C18-Stage-tips [35] for LC-MS/MS analysis. LC-MS/MS analysis was performed using Orbitrap Fusion Lumos (Thermo Fisher Scientific) with a "high/high" acquisition strategy. The peptide separation was carried out on an EASY-Spray column (50 cm × $75 \mu$ m i.d., PepMap C18, $2 \mu$ m particles, $100 \AA ̊$ pore size, Thermo Fisher Scientific). Mobile phase A consisted of water and $0.1 \% \mathrm{v} / \mathrm{v}$ formic acid. Mobile phase $B$ consisted of $80 \% \mathrm{v} / \mathrm{v}$ acetonitrile and $0.1 \% \mathrm{v} / \mathrm{v}$ formic acid. Peptides were loaded at a flow rate of $0.3 \mu \mathrm{l} / \mathrm{min}$ and eluted at $0.2 \mu \mathrm{l} / \mathrm{min}$ using a linear gradient going from $2 \%$ mobile phase $B$ to $40 \%$ mobile phase B over 109 or 139 min (each sample has been running three time with different gradients), followed by a linear increase from $40 \%$ to $95 \%$ mobile phase $\mathrm{B}$ in $11 \mathrm{~min}$. The eluted peptides were directly introduced into the mass spectrometer. MS data were acquired in the data-dependent mode with $3 \mathrm{~s}$ acquisition cycle. Precursor spectra were recorded in the Orbitrap with a resolution of 120,000 . The ions with a precursor charge state between $3+$ and $8+$ were isolated with a window size of $1.6 \mathrm{~m} / \mathrm{z}$ and fragmented using high-energy collision dissociation (HCD) with collision energy 30 . The fragmentation spectra were recorded in the Orbitrap with a resolution of 15,000. Dynamic exclusion was enabled with single repeat count and $60 \mathrm{~s}$ exclusion duration. The mass spectrometric raw files were processed into peak lists using ProteoWizard (version 3.0.6618) [36], and cross-linked peptides were matched to spectra using Xi software (version 1.6.743) [37] with in-search assignment of monoisotopic peaks [38]. Search parameters were MS accuracy, 3 ppm; MS/MS accuracy, 10ppm; enzyme, trypsin; cross-linker, EDC; max missed cleavages, 4; missing mono-isotopic peaks, 2; fixed modification, carbamidomethylation on cysteine; variable modifications, oxidation on methionine and phosphorylation on threonine for phosphorylated sample; fragments, $b$ and $y$ ions with loss of $\mathrm{H} 2 \mathrm{O}, \mathrm{NH} 3$ and $\mathrm{CH} 3 \mathrm{SOH}$. FDR was computed using XiFDR and results reported at 5\% residue level FDR [39].

\section{References}

1. Yamagishi, Y., Honda, T., Tanno, Y., and Watanabe, Y. (2010). Two Histone Marks Establish the Inner Centromere and Chromosome Bi-Orientation. Science 330, 239 LP - 243. 
2. Kelly, A.E., Ghenoiu, C., Xue, J.Z., Zierhut, C., Kimura, H., and Funabiki, H. (2010). Survivin Reads Phosphorylated Histone H3 Threonine 3 to Activate the Mitotic Kinase Aurora B. Science 330, 235 LP - 239.

3. Wang, F., Dai, J., Daum, J.R., Niedzialkowska, E., Banerjee, B., Stukenberg, P.T., Gorbsky, G.J., and Higgins, J.M.G. (2010). Histone H3 Thr-3 Phosphorylation by Haspin Positions Aurora B at Centromeres in Mitosis. Science 330, 231 LP - 235.

4. Funabiki, H., and Wynne, D.J. (2013). Making an effective switch at the kinetochore by phosphorylation and dephosphorylation. Chromosoma 122, 135-158.

5. Gelens, L., Qian, J., Bollen, M., and Saurin, A.T. (2018). The Importance of KinasePhosphatase Integration: Lessons from Mitosis. Trends in Cell Biology 28, 6-21.

6. Saurin, A.T. (2018). Kinase and Phosphatase Cross-Talk at the Kinetochore In Frontiers in Cell and Developmental Biology Volume 6 p. 62.

7. Carmena, M., Wheelock, M., Funabiki, H., and Earnshaw, W.C. (2012). The chromosomal passenger complex (CPC): from easy rider to the godfather of mitosis. Nature Reviews Molecular Cell Biology 13, 789.

8. Hindriksen, S., Lens, S.M.A., and Hadders, M.A. (2017). The Ins and Outs of Aurora B Inner Centromere Localization. Frontiers in cell and developmental biology 5, 112.

9. Klein, U.R., Nigg, E.A., and Gruneberg, U. (2006). Centromere targeting of the chromosomal passenger complex requires a ternary subcomplex of Borealin, Survivin, and the N-terminal domain of INCENP. Molecular biology of the cell 17, 2547-2558.

10. Jeyaprakash, A.A., Klein, U.R., Lindner, D., Ebert, J., Nigg, E.A., and Conti, E. (2007). Structure of a Survivin-Borealin-INCENP Core Complex Reveals How Chromosomal Passengers Travel Together. Cell 131, 271-285.

11. Bishop, J.D., and Schumacher, J.M. (2002). Phosphorylation of the carboxyl terminus of inner centromere protein (INCENP) by the Aurora B Kinase stimulates Aurora B kinase activity. The Journal of biological chemistry 277, 27577-27580.

12. Honda, R., Körner, R., and Nigg, E.A. (2003). Exploring the functional interactions between Aurora B, INCENP, and survivin in mitosis. Molecular biology of the cell 14, 3325-3341.

13. Sessa, F., Mapelli, M., Ciferri, C., Tarricone, C., Areces, L.B., Schneider, T.R., Stukenberg, P.T., and Musacchio, A. (2005). Mechanism of Aurora B Activation by INCENP and Inhibition by Hesperadin. Molecular Cell 18, 379-391.

14. Lipp, J.J., Hirota, T., Poser, I., and Peters, J.-M. (2007). Aurora B controls the association of condensin I but not condensin II with mitotic chromosomes. Journal of Cell Science 120, 1245 LP - 1255.

15. Takemoto, A., Murayama, A., Katano, M., Urano, T., Furukawa, K., Yokoyama, S., Yanagisawa, J., Hanaoka, F., and Kimura, K. (2007). Analysis of the role of Aurora B on the chromosomal targeting of condensin I. Nucleic acids research 35, 2403-2412.

16. Cooke, C.A., Heck, M.M., and Earnshaw, W.C. (1987). The inner centromere protein (INCENP) antigens: movement from inner centromere to midbody during mitosis. The Journal of cell biology 105, 2053-2067.

17. Adams, R.R., Wheatleya, S.P., Gouldsworthy, A.M., Kandels-Lewis, S.E., Carmena, M., Smythe, C., Gerloff, D.L., and Earnshaw, W.C. (2000). INCENP binds the Aurorarelated kinase AIRK2 and is required to target it to chromosomes, the central spindle and cleavage furrow. Current Biology 10, 1075-1078. 
18. Uren, A.G., Wong, L., Pakusch, M., Fowler, K.J., Burrows, F.J., Vaux, D.L., and Choo, K.H.A. (2000). Survivin and the inner centromere protein INCENP show similar cellcycle localization and gene knockout phenotype. Current Biology 10, 1319-1328.

19. Gassmann, R., Carvalho, A., Henzing, A.J., Ruchaud, S., Hudson, D.F., Honda, R., Nigg, E.A., Gerloff, D.L., and Earnshaw, W.C. (2004). Borealin: a novel chromosomal passenger required for stability of the bipolar mitotic spindle. The Journal of cell biology 166, 179-191.

20. Trivedi, P., and Stukenberg, P.T. (2016). A Centromere-Signaling Network Underlies the Coordination among Mitotic Events. Trends in biochemical sciences 41, 160-174.

21. Tsukahara, T., Tanno, Y., and Watanabe, Y. (2010). Phosphorylation of the CPC by Cdk1 promotes chromosome bi-orientation. Nature 467, 719.

22. Barbera, A.J., Chodaparambil, J.V., Kelley-Clarke, B., Joukov, V., Walter, J.C., Luger, K., and Kaye, K.M. (2006). The Nucleosomal Surface as a Docking Station for Kaposi's Sarcoma Herpesvirus LANA. Science 311, 856 LP - 861.

23. Roussel, L., Erard, M., Cayrol, C., and Girard, J.-P. (2008). Molecular mimicry between IL-33 and KSHV for attachment to chromatin through the H2A-H2B acidic pocket. EMBO reports 9, 1006-1012.

24. Makde, R.D., England, J.R., Yennawar, H.P., and Tan, S. (2010). Structure of RCC1 chromatin factor bound to the nucleosome core particle. Nature 467, 562.

25. Armache, K.-J., Garlick, J.D., Canzio, D., Narlikar, G.J., and Kingston, R.E. (2011). Structural Basis of Silencing: Sir3 BAH Domain in Complex with a Nucleosome at 3.0 Å Resolution. Science 334, 977 LP - 982.

26. Kato, H., van Ingen, H., Zhou, B.-R., Feng, H., Bustin, M., Kay, L.E., and Bai, Y. (2011). Architecture of the high mobility group nucleosomal protein 2-nucleosome complex as revealed by methyl-based NMR. Proceedings of the National Academy of Sciences of the United States of America 108, 12283-12288.

27. Ruppert, J.G., Samejima, K., Platani, M., Molina, O., Kimura, H., Jeyaprakash, A.A., Ohta, S., and Earnshaw, W.C. (2018). HP1 $\alpha$ targets the chromosomal passenger complex for activation at heterochromatin before mitotic entry. The EMBO journal 37, e97677.

28. Earnshaw, W.C., and Cooke, C.A. (1991). Analysis of the distribution of the INCENPs throughout mitosis reveals the existence of a pathway of structural changes in the chromosomes during metaphase and early events in cleavage furrow formation. Journal of Cell Science 98, 443 LP - 461.

29. Cai, Y., Hossain, M.J., Hériché, J.-K., Politi, A.Z., Walther, N., Koch, B., Wachsmuth, M., Nijmeijer, B., Kueblbeck, M., Martinic-Kavur, M., et al. (2018). Experimental and computational framework for a dynamic protein atlas of human cell division. Nature 561, 411-415.

30. Davey, C.A., Sargent, D.F., Luger, K., Maeder, A.W., and Richmond, T.J. (2002). Solvent Mediated Interactions in the Structure of the Nucleosome Core Particle at 1.9Å Resolution. Journal of Molecular Biology 319, 1097-1113.

31. Bourhis, E., Lingel, A., Phung, Q., Fairbrother, W.J., and Cochran, A.G. (2009). Phosphorylation of a borealin dimerization domain is required for proper chromosome segregation. Biochemistry 48, 6783-6793.

32. Luger, K., Rechsteiner, T.J., and Richmond, T.J. (1999). Expression and Purification of Recombinant Histones and Nucleosome Reconstitution BT - Chromatin Protocols. P.B. Becker, ed. (Totowa, NJ: Humana Press), pp. 1-16. 
33. Elbashir, S.M., Harborth, J., Lendeckel, W., Yalcin, A., Weber, K., and Tuschl, T. (2001). Duplexes of 21-nucleotide RNAs mediate RNA interference in cultured mammalian cells. Nature 411, 494.

34. Dai, J., Sultan, S., Taylor, S.S., and Higgins, J.M.G. (2005). The kinase haspin is required for mitotic histone $\mathrm{H} 3 \mathrm{Thr} 3$ phosphorylation and normal metaphase chromosome alignment. Genes \& development 19, 472-488.

35. Rappsilber, J., Mann, M., and Ishihama, Y. (2007). Protocol for micro-purification, enrichment, pre-fractionation and storage of peptides for proteomics using StageTips. Nature Protocols 2, 1896.

36. Kessner, D., Chambers, M., Burke, R., Agus, D., and Mallick, P. (2008). ProteoWizard: open source software for rapid proteomics tools development. Bioinformatics (Oxford, England) 24, 2534-2536.

37. Mendes, M.L., Fischer, L., Chen, Z.A., Barbon, M., O\&\#039;Reilly, F.J., BohlkeSchneider, M., Belsom, A., Dau, T., Combe, C.W., Graham, M., et al. (2018). An integrated workflow for cross-linking/mass spectrometry. bioRxiv, 355396.

38. Lenz, S., Giese, S.H., Fischer, L., and Rappsilber, J. (2018). In-Search Assignment of Monoisotopic Peaks Improves the Identification of Cross-Linked Peptides. Journal of proteome research 17, 3923-3931.

39. Fischer, L., and Rappsilber, J. (2017). Quirks of Error Estimation in Cross-Linking/Mass Spectrometry. Analytical chemistry 89, 3829-3833. 